\title{
Degree of entanglement for two qubits
}

\author{
Jing-Ling Chen, ${ }^{1,2} *$ Libin Fu, ${ }^{1}$ Abraham A. Ungar, ${ }^{3}{ }^{\dagger}$ and Xian-Geng Zhao ${ }^{1}$ \\ 1 Laboratory of Computational Physics, \\ Institute of Applied Physics and Computational Mathematics, \\ P.O. Box 8009(26), Beijing 100088, People's Republic of China. \\ 2 Department of Physics, Faculty of Science, National University of Singapore, \\ Lower Kent Ridge, Singapore 119260, Republic of Singapore. \\ ${ }^{3}$ Department of Mathematics, North Dakota State University, Fargo, North Dakota 58105, USA.
}

\begin{abstract}
In this paper, we present a measure to quantify the degree of entanglement for two qubits in a pure state.
\end{abstract}

03.67.-a, 03.65.Ud

\section{INTRODUCTION}

Quantum entanglement is the most surprising nonclassical property of composite quantum systems [1]. As it is well-known, a qubit (or a spin-1/2 particle) is described by the $2 \times 2$ density matrix $\rho(\mathbf{n})=$ $(\mathbf{1}+\vec{\sigma} \cdot \mathbf{n}) / 2,|\mathbf{n}| \leq 1$, where $\mathbf{1}$ is the unit matrix, $\vec{\sigma}=\left(\sigma_{1}, \sigma_{2}, \sigma_{3}\right)$ the Pauli matrices vector, and $\mathbf{n}$ the Bloch vector. $|\mathbf{n}|=1$ corresponds to a pure state, otherwise a mixed state. Whereas, an entangled pairs of two qubits is completely described by the following $4 \times 4$ density matrix:

$$
\rho_{A B}=\frac{1}{4}\left(\mathbf{1} \otimes \mathbf{1}+\vec{\sigma}^{A} \cdot \mathbf{u} \otimes \mathbf{1}+\mathbf{1} \otimes \vec{\sigma}^{B} \cdot \mathbf{v}+\sum_{i, j=1}^{3} \beta_{i j} \sigma_{i}^{A} \otimes \sigma_{j}^{B}\right)
$$

from which one could obtain two reduced density matrices

$$
\begin{aligned}
& \rho_{A}=\operatorname{tr}_{B}\left(\rho_{A B}\right)=\frac{1}{2}\left(\mathbf{1}+\vec{\sigma}^{A} \cdot \mathbf{u}\right), \\
& \rho_{B}=\operatorname{tr}_{A}\left(\rho_{A B}\right)=\frac{1}{2}\left(\mathbf{1}+\vec{\sigma}^{B} \cdot \mathbf{v}\right),
\end{aligned}
$$

for the two qubits $\mathrm{A}$ and $\mathrm{B}$, where $\mathbf{u}$ and $\mathbf{v}$ are Bloch vectors for particles $\mathrm{A}$ and $\mathrm{B}$, respectively; $\beta_{i j}$ are some real numbers.

It has been shown that entangled pairs are a more powerful resource than separable, i.e., disentangled, pairs in a number of applications, such as quantum cryptography [2], dense coding [3], teleportation [1] and investigations of quantum channels 胞, communication protocols and computation [6] [7]. The superior potentiality of entangled states has raised a natural question: "How much are two particles entangled?", since pairs with a high degree of entanglement should be a better resource than less entangled ones. Many measures of entanglement proposed in the past have relied on either the Schmidt decomposition [8] or decomposition in a magic basis [9]. In an interesting paper, Abouraddy et al. devised a new measure of entanglement for pure bipartite states of two qubits, based on a decomposition of the state vector as a superposition of a maximally entangled state vector and an orthogonal factorizable one [10]. Although there are many such decompositions, the weights of the two superposed states are remarkably unique. The square of the weight of the maximally entangled state vector (i.e., $P_{E}=p^{2}$ ) is then defined as the degree of

\footnotetext{
*Email address: jinglingchen@eyou.com

${ }^{\dagger}$ Email address: ungar@gyro.math.ndsu.nodak.edu
} 
entanglement for two qubits, such a measure is consistent with three measures of entanglement previously set forth: maximal violation of Bell's inequality [11], concurrence [9] and two-particle visibility [12].

The purpose of this paper is to propose a new approach to the problem of defining the degree of entanglement for two qubits in a pure state. In Sec. II, a new measure is formulated to quantify the degree of entanglement. Some examples are given in Sec. III. Conclusion and discussion are made in the last section.

\section{FORMALISM}

Theorem: If $\rho_{A B}$ is a pure state, then its degree of entanglement $P_{E}$ is equal to

$$
P_{E}=(-\operatorname{det} \hat{\alpha})^{1 / 4}
$$

where the matrix $\hat{\alpha}$ is

$$
\hat{\alpha}=\left(\begin{array}{llll}
1 & v_{1} & v_{2} & v_{3} \\
u_{1} & \beta_{11} & \beta_{12} & \beta_{13} \\
u_{2} & \beta_{21} & \beta_{22} & \beta_{23} \\
u_{3} & \beta_{31} & \beta_{32} & \beta_{33}
\end{array}\right) .
$$

Proof: $\rho_{A B}$ is a pure state implies that $\rho_{A B}^{2}=\rho_{A B}$, from which one obtains the following constraints among $u_{i}, v_{i}$ and $\beta_{i j}(i, j=1,2,3)$ :

$$
\begin{gathered}
u_{i}=\beta_{i 1} v_{1}+\beta_{i 2} v_{2}+\beta_{i 3} v_{3}, \\
v_{i}=\beta_{1 i} u_{1}+\beta_{2 i} u_{2}+\beta_{3 i} u_{3}, \\
\sum_{i, j} \beta_{i j}^{2}=3-|\mathbf{u}|^{2}-|\mathbf{v}|^{2} \\
\beta_{i j}=u_{i} v_{j}-(-1)^{i+j} M_{i j},
\end{gathered}
$$

where $M_{i j}$ is the algebraic complement of the matrix element $\beta_{i j}$ for the following $\hat{\beta}$ matrix:

$$
\hat{\beta}=\left(\begin{array}{lll}
\beta_{11} & \beta_{12} & \beta_{13} \\
\beta_{21} & \beta_{22} & \beta_{23} \\
\beta_{31} & \beta_{32} & \beta_{33}
\end{array}\right) .
$$

Eqs. (5) and (6) can be recast as $\hat{\beta} \mathbf{v}=\mathbf{u}, \hat{\beta}^{T} \mathbf{u}=\mathbf{v}$, where $T$ represents transpose and $\mathbf{u}=\left(u_{1}, u_{2}, u_{3}\right)^{T}$. An interesting result, i.e., $|\mathbf{u}|=|\mathbf{v}|$, will be obtained immediately from Eqs. (5) and (6) for the pure state $\rho_{A B}[13]$. From Eq. (8) we have

$$
\begin{aligned}
\beta_{11}^{2}+\beta_{12}^{2}+\beta_{13}^{2} & =\beta_{11} u_{1} v_{1}+\beta_{12} u_{1} v_{2}+\beta_{13} u_{1} v_{3} \\
& -\left[(-1)^{1+1} \beta_{11} M_{11}+(-1)^{1+2} \beta_{12} M_{12}+(-1)^{1+3} \beta_{13} M_{13}\right] .
\end{aligned}
$$

Due to $\operatorname{det} \hat{\beta}=\beta_{11} M_{11}-\beta_{12} M_{12}+\beta_{13} M_{13}$ and Eq. (5), one obtains

$$
\beta_{11}^{2}+\beta_{12}^{2}+\beta_{13}^{2}-u_{1}^{2}=-\operatorname{det} \hat{\beta} .
$$

Similarly, 


$$
\begin{aligned}
& \beta_{21}^{2}+\beta_{22}^{2}+\beta_{23}^{2}-u_{2}^{2}=-\operatorname{det} \hat{\beta}, \\
& \beta_{31}^{2}+\beta_{32}^{2}+\beta_{33}^{2}-u_{3}^{2}=-\operatorname{det} \hat{\beta} .
\end{aligned}
$$

After combining Eqs. (7), (11), (12), and taking $|\mathbf{u}|=|\mathbf{v}|$ into account, one easily obtains $-\operatorname{det} \hat{\beta}=1-|\mathbf{u}|^{2}$ 13. Consequently, we have

$$
(-\operatorname{det} \hat{\alpha})^{1 / 4}=\left[(-\operatorname{det} \hat{\beta})\left(1-|\mathbf{u}|^{2}\right)\right]^{1 / 4}=\sqrt{1-|\mathbf{u}|^{2}} .
$$

One can know from Ref. 10] that $P_{E}=2 \kappa_{1} \kappa_{2}$, where $\kappa_{1}$ and $\kappa_{2}$ are the two coefficients in the Schmidt decomposition $|\Psi\rangle=\kappa_{1}\left|x_{1}, y_{1}\right\rangle+\kappa_{2}\left|x_{2}, y_{2}\right\rangle, \rho_{A B}=|\Psi\rangle\langle\Psi|$, where $\left\{\left|x_{1}\right\rangle,\left|x_{2}\right\rangle\right\}$ and $\left\{\left|y_{1}\right\rangle,\left|y_{2}\right\rangle\right\}$ are orthogonal bases for the Hilbert spaces of particles $A$ and $B$, respectively. It is easy to prove that $\kappa_{1}=\sqrt{(1+|\mathbf{u}|) / 2}, \kappa_{2}=\sqrt{(1-|\mathbf{u}|) / 2}$, which are square-roots of the two eigenvalues of the reduced matrix $\rho_{A}$ or $\rho_{B}$. Therefore we have $P_{E}=(-\operatorname{det} \hat{\alpha})^{1 / 4}$. This ends the proof.

\section{EXAMPLES}

Example 1. For the state $|\Psi\rangle=(|00\rangle+|01\rangle+|11\rangle) / \sqrt{3}$, one obtains the density matrix

$$
\rho_{A B}=|\Psi\rangle\langle\Psi|=\frac{1}{3}\left(\begin{array}{llll}
1 & 1 & 0 & 1 \\
1 & 1 & 0 & 1 \\
0 & 0 & 0 & 0 \\
1 & 1 & 0 & 1
\end{array}\right)
$$

with the Bloch vectors $\mathbf{u}=(2 / 3,0,1 / 3)^{T}, \mathbf{v}=(2 / 3,0,-1 / 3)^{T}$, and the alpha matrix

$$
\hat{\alpha}=\frac{1}{3}\left(\begin{array}{cccc}
3 & 2 & 0 & -1 \\
2 & 2 & 0 & -2 \\
0 & 0 & -2 & 0 \\
1 & 2 & 0 & 1
\end{array}\right) .
$$

One can have $P_{E}=2 / 3$, which is consistent with the result in Ref. [10].

Example 2. For the state $|\Psi\rangle=[|00\rangle+2(|01\rangle+|11\rangle)] / 3$, the density matrix is

$$
\rho_{A B}=|\Psi\rangle\langle\Psi|=\frac{1}{9}\left(\begin{array}{llll}
1 & 2 & 0 & 2 \\
2 & 4 & 0 & 4 \\
0 & 0 & 0 & 0 \\
2 & 4 & 0 & 4
\end{array}\right)
$$

with $\mathbf{u}=(8 / 9,0,1 / 9)^{T}, \mathbf{v}=(4 / 9,0,-7 / 9)^{T}$, and the alpha matrix

$$
\hat{\alpha}=\frac{1}{9}\left(\begin{array}{cccc}
9 & 4 & 0 & -7 \\
8 & 4 & 0 & -8 \\
0 & 0 & -4 & 0 \\
1 & 4 & 0 & 1
\end{array}\right) .
$$

Hence the degree of entanglement is $P_{E}=4 / 9$.

Example 3. For the maximally entangled state $|\Psi\rangle=(|00\rangle+|11\rangle) / \sqrt{2}$, one obtains the density matrix

$$
\rho_{A B}=|\Psi\rangle\langle\Psi|=\frac{1}{2}\left(\begin{array}{llll}
1 & 0 & 0 & 1 \\
0 & 0 & 0 & 0 \\
0 & 0 & 0 & 0 \\
1 & 0 & 0 & 1
\end{array}\right)
$$


with the Bloch vectors $\mathbf{u}=\mathbf{v}=(0,0,0)^{T}$, and the alpha matrix

$$
\hat{\alpha}=\left(\begin{array}{cccc}
1 & 0 & 0 & 0 \\
0 & 1 & 0 & 0 \\
0 & 0 & -1 & 0 \\
0 & 0 & 0 & 1
\end{array}\right)
$$

Thus $P_{E}=1$ reaches the highest value.

Example 4. For the disentangled pure state $\rho_{A B}=\frac{1}{2}\left(\mathbf{1}+\vec{\sigma}^{A} \cdot \mathbf{u}\right) \otimes \frac{1}{2}\left(\mathbf{1}+\vec{\sigma}^{B} \cdot \mathbf{v}\right)$, where $|\mathbf{u}|=|\mathbf{v}|=1$, we have the alpha matrix as

$$
\hat{\alpha}=\left(\begin{array}{cccc}
1 & v_{1} & v_{2} & v_{3} \\
u_{1} & u_{1} v_{1} & u_{1} v_{2} & u_{1} v_{3} \\
u_{2} & u_{2} v_{1} & u_{2} v_{2} & u_{2} v_{3} \\
u_{3} & u_{3} v_{1} & u_{3} v_{2} & u_{3} v_{3}
\end{array}\right)
$$

Obviously $P_{E}=0$ indicates that $\rho_{A B}$ is disentangled.

\section{CONCLUSION AND DISCUSSION}

In conclusion, we have presented a measure to quantify the degree of entanglement for two qubits in a pure state. We would like to make some discussion in the following:

(i) The similar idea developed in this paper could be generalized to quantify the degree of entanglement for two qu $N$ its (i.e., $N$-state quantum systems, $N=2$ and $N=3$ correspond to a qubit and a qutrit, respectively) [14] 15] in a pure state. For instance, the density matrix for two entangled qutrits could be written as

$$
\rho_{A B}=\frac{1}{9}\left(\mathbf{1} \otimes \mathbf{1}+\sqrt{3} \vec{\lambda}^{A} \cdot \mathbf{u} \otimes \mathbf{1}+\sqrt{3} \mathbf{1} \otimes \vec{\lambda}^{B} \cdot \mathbf{v}+\frac{3}{2} \sum_{i, j=1}^{8} \beta_{i j} \lambda_{i}^{A} \otimes \lambda_{j}^{B}\right),
$$

where $\lambda_{i}(i=1,2, \ldots, 8)$ are the eight hermitian generators of $S U(3)$ (namely the usual Gell-mann matrices). For the state of two entangled qutrits

$$
|\Psi\rangle=\frac{1}{3}(|00\rangle+|11\rangle+|22\rangle),
$$

its corresponding density matrix is $[15]$

$$
\rho_{A B}=\frac{1}{9}\left(\mathbf{1} \otimes \mathbf{1}+\frac{3}{2} \sum_{i, j=1}^{8} \beta_{i j} \lambda_{i}^{A} \otimes \lambda_{j}^{B}\right),
$$

with the non-zero coefficients $\beta_{11}=\beta_{33}=\beta_{44}=\beta_{66}=\beta_{88}=1, \beta_{22}=\beta_{55}=\beta_{77}=-1$. The elements $\beta_{i j}, 1, \mathbf{u}$ and $\mathbf{v}$ form a $9 \times 9$ matrix $\hat{\alpha}$, it is easy to show that $P_{E}=(-\operatorname{det} \hat{\alpha})^{1 / 4}=1$, which indicates that the state $|\Psi\rangle$ in Eq. (19) is just a maximally entangled state.

(ii) After making the parametrization $\mathbf{u}=\hat{\mathbf{u}} \tanh \phi_{\mathbf{u}}$, where $\hat{\mathbf{u}}=\mathbf{u} /|\mathbf{u}|$, the density matrix of a qubit $\rho(\mathbf{u})=(\mathbf{1}+\vec{\sigma} \cdot \mathbf{u}) / 2$ can be connected to the Lorentz boost matrix $L(\mathbf{u})=\exp \left(\varphi_{\mathbf{u}} \vec{\sigma} \cdot \hat{\mathbf{u}} / 2\right)=$ $\mathbf{1} \cosh \left(\varphi_{\mathbf{u}} / 2\right)+\vec{\sigma} \cdot \hat{\mathbf{u}} \sinh \left(\varphi_{\mathbf{u}} / 2\right)$ as 16

$$
\rho(\mathbf{u})=\frac{L(\mathbf{u})}{2 \cosh \phi_{\mathbf{u}}}, \quad \phi_{\mathbf{u}}=\varphi_{\mathbf{u}} / 2 .
$$

Obviously, $\rho(\mathbf{u})$ and $L(\mathbf{u})$ are in one-to-one correspondence. For the former, the physical meaning of the vector $\mathbf{u}$ is the Bloch vector in quantum mechanics, while for the latter the relativistic velocity. Due 
to the rapidity $\varphi$, i.e., the hyperbolic angle, special relativity can be formulated in terms of hyperbolic geometry. As a result, some physical quantities have been found to have geometric significance, such as the Thomas rotation angle corresponds to the defect of a hyperbolic triangle [17] [18]. After viewing the Bloch vector $\mathbf{u}$ as an analogous relativistic velocity, the Bures fidelity $\left.F\left(\rho_{1}, \rho_{2}\right)=\operatorname{tr} \sqrt{\sqrt{\rho_{1}} \rho_{2} \sqrt{\rho_{1}}}\right]^{2}$ was found to have a geometric interpretation in the framework of hyperbolic geometry [16]. Similarly, with the aid of the parametrization $\mathbf{u}=\hat{\mathbf{u}} \tanh \phi_{\mathbf{u}}$, it is not difficult to find that the entanglement degree $P_{E}=\sqrt{1-|\mathbf{u}|^{2}}=1 / \cosh \phi_{\mathbf{u}}$ for two qubits in a pure state is the reciprocal of the Lorentz factor [18] in the hyperbolic geometry. The extension of our approach to the mixed states of two entangled qubits will be discussed elsewhere.

[1] E. Schrödinger, Naturwissenschafen 23, 807 (1935); 23, 823 (1935); 23, 844 (1935); A. Einstein, B. Podolsky and N. Rosen, Phys. Rev. 47, 777 (1935); J.S. Bell, Physics (Long Island, NY), 1, 195 (164); reprinted in J.S. Bell, Speakable and Unspeakable in Quantum Mechanics, (Cambridge University Press, Cambridge, 1987).

[2] A.K. Ekert, Phys. Rev. Lett. 67, 661 (1991).

[3] C.H. Bennett and S.J. Wiesner, Phys. Rev. Lett. 69, 2881 (1992).

[4] The theoretical proposals are in C.H. Bennnett et al., Phys. Rev. Lett. 70, 1895 (1993); L. Davidovich et al., Phys. Rev. A 50, R895 (1994). For the experiments see D. Boschi et al., Phys. Rev. Lett. 80, 1121 (1998); A. Furusawa et al., Science 282, 706 (1998); M.A. Nielsen, E. Knill and R. Laflamme, Nature 396, 52 (1998).

[5] D.G. Fischer, H. Mack, M.A. Cirone and M. Freyberger, Phys. Rev. A 64, 022309 (2001); C. Machiavello and G.M. Palma, e-print quant-ph/0107052.

[6] H.-K. Lo, T. Spiller and S. Popescu (eds.), Introduction to Quantum Computation and Information (World Scientific Publishing, Singapore, 1998); J. Gruska, Quantum Computing (McGraw-Hill, London, 1999); G. Alber et al., Quantum Information: An Introduction to Basic Theoretical Concepts and Experiments (Springer, Berlin, 2001).

[7] M.A. Nielsen and I.L. Chuang, Quantum Computation and Quantum Information (Cambridge University Press, 2000); D. Bouwmeester, A. Ekert and A. Zeilinger (eds.), The Physics of Quantum Information (Springer, Berlin, 2000).

[8] A. Peres, Quantum Theory: Concepts and Methods (Kluwer, The Netherlands, 1993); M.A. Cirone, e-print quant-ph/0110139.

[9] S. Hill and W.K. Wootters, Phys. Rev. Lett. 78, 5022 (1997); W.K. Wootters, Phys. Rev. Lett. 80, 2245 (1998); K.M. O'Connor and W.K. Wootters, Phys. Rev. A 63, 052302 (2001).

[10] A.F. Abouraddy, B.E.A. Saleh, A.V. Sergienko and M.C. Teich, Phys. Rev. A 64, 050101(R) (2001); e-print quant-ph/0109081.

[11] J.F. Clauser, M.A. Horne, A. Shimony and R.A. Holt, Phys. Rev. Lett. 23, 880 (1969); N. Gisin, Phys. Lett. A 154, 201 (1991); S. Popescu and D. Rohrlich, Phys. Lett. A 166, 293 (1992).

[12] G. Jaeger, A. Shimony and L. Vaidman, Phys. Rev. A 51, 54 (1993).

[13] H.J Kummer, Inter. J. Theore. Phys. 40, 1071 (2001).

[14] D. Kaszlikowski, P. Gnaciński, M. Żukowski, W. Miklaszewski and A. Zeilinger, Phys. Rev. Lett. 85, 4418 (2000); J.-L. Chen, D. Kaszlikowski, L.C. Kwek, M. Żukowski, and C. H. Oh, Phys. Rev. A 64, 052109 (2001).

[15] C.M. Caves and G.J. Milburn, e-print quant-ph/9910001; P. Rungta, W.J. Munro, K. Nemoto, P. Deuar, G.J. Milburn and C.M. Caves, e-print quant-ph/0001075.

[16] J.-L. Chen, L. Fu, A.A. Ungar and X.-G. Zhao, Geometric observation for the Bures fidelity between two states of a qubit, Phys. Rev. A, 65, 024303 (2001).

[17] J.-L. Chen and M.-L. Ge, J. Geom. Phys. 25, 341 (1998); P.K. Aravind, Am. J. Phys. 65, 634 (1997); A.A. Ungar, Found. Phys. 27, 881 (1997); J.-L. Chen and A.A. Ungar, Found. Phys. 31, 1611 (2001).

[18] Abraham A. Ungar, Beyond the Einstein addition law and its gyroscopic Thomas precession: the theory of gyrogroups and gyrovector spaces (Kluwer Academic Publishers, Dordrecht, 2001). 